higher or lower concentrations in the PsA group compared to the control or the RA group. We selected two of these significant metabolites to build a classification model based on the linear support vector machine (SVM) method, and the area-under-the-curve (AUC) value of the resulting receiver operating characteristic (ROC) curve was 0.929 (95\% confidence interval: 0.899-0.956). Similarly, 37 metabolites could differentiate AS samples from RAs and controls. A proposed diagnostic panel containing four metabolites demonstrated an AUC value of 0.890 (0.843-0.934). For the last step, distinguishing between PsA and AS, there were 15 significantly increased metabolites and 9 lowered ones. The biomarker panel consisting of the top three metabolites also achieved good discriminatory power with $\mathrm{AUC}=0.827$ (0.717-0.919).

Conclusion: Isotope-labeling-LC-MS-based metabolomics has revealed biomarker candidates that can specifically differentiate PsA or AS patients from control populations.

Disclosure of Interests: Wei Han: None declared, Xiaohang Wang: None declared, Liang Li: None declared, Stephanie Wichuk: None declared, Edna Hutchings: None declared, Rana Dadashova: None declared, Joel Paschke: None declared, Walter P Maksymowych Grant/research support from: Received research and/or educational grants from Abbvie, Novartis, Pfizer, UCB, Consultant of: WPM is Chief Medical Officer of CARE Arthritis Limited, has received consultant/participated in advisory boards for Abbvie, Boehringer, Celgene, Eli-Lilly, Galapagos, Gilead, Janssen, Novartis, Pfizer, UCB, Speakers bureau: Received speaker fees from Abbvie, Janssen, Novartis, Pfizer, UCB.

DOI: 10.1136/annrheumdis-2020-eular.5912

\section{OP0305 RHO-GTPASE PATHWAYS MAY DIFFERENTIATE RESPONDER AND NON-RESPONDERS TO TUMOUR NECROSIS FACTOR INHIBITOR (TNFI) AND INTERLEUKIN-17A INHIBITOR (IL-17AI) THERAPY IN PSORIATIC ARTHRITIS (PSA)}

S. Rahmati ${ }^{1}$, D. O'rielly ${ }^{2}$, Q. LI ${ }^{1}$, D. Codner ${ }^{2}$, A. Dohey ${ }^{2}$, K. Jenkins ${ }^{2}$, I. Jurisica ${ }^{3}$ D. D. Gladman ${ }^{1}$, V. Chandran ${ }^{1}$, P. Rahman ${ }^{2} .{ }^{1}$ University of Toronto, Toronto,

Canada; ${ }^{2}$ Memorial University, St. John's, Canada; ${ }^{3}$ Krembil Research Institute, Toronto, Canada

Background: In PsA there is a pressing need to develop a coherent strategy for identifying initial and subsequent biologic responders. PsA patients present substantial heterogeneity in response to biologics, and molecular subtyping will help to identify the right patient for the right treatment.

Objectives: To identify transcript profiles (biomarkers) that will select TNFi and IL-17Ai responders in PsA using baseline CD4+ cells; and elucidate novel signaling pathways relevant to biologic disease modifying antirheumatic drug (bDMARD) response using a systems biology approach.

Methods: Consenting patients initiating TNFi agents (20 patients) or IL-17Ai agents (20 patients) with moderate-to-severe PsA were assessed with a comprehensive standardized protocol at baseline and at 3 months. Responder to bDMARDs was defined by Disease Activity index for PSoriatic Arthritis (DAPSA) score of less than 14 (low disease activity). Global transcript profiling was performed on all patients prior to initiation of and 3 months post bDMARDs. We mapped RNA-seq reads to the hg19 reference genome using STAR and quantified transcripts with Cufflinks. The transcripts per million (TPM) values were log-transformed for statistical analyses

Results: The demographics of PsA patients for both treatment groups are presented (Table 1). Differentially expressed genes (DEGs) were identified using the limma tool for TNFi and IL-17Ai responders and non-responders (Figure 1) as well as DEGs that differentiated TNFi from IL-17Ai response and non-response. Integration of differential gene expression data with tissue-specific protein-protein interactions (IID version 2018-11) identified 117 and 132 DEGs between responders and non-responders to TNFi and IL-17Ai treatments, respectively. Comprehensive pathway enrichment analysis of these genes using pathDIP (v 4.1) revealed 576 (out of 5380) pathways enriched in 117 DEGs between responders and non-responders to $\mathrm{TNFi}$, and 125 pathways enriched in 132 DEGs between responders and non-responders to IL-17Ai. Interestingly, while these two gene lists share only 17 genes, they have 79 enriched pathways in common suggesting potential effect of two different treatments on similar pathways but through different pathway members (Figure 2). Moreover, it suggests potential importance of the 17 shared genes in association with these pathways. Most of these pathways are related to innate and adaptive immune system, and to "osteoclast differentiation". Among 46 pathways specific to response to IL-17Ai, multiple Rho-GTPase-related pathways were identified. It has been shown that experimental inhibition of ROCK2, a target of Rho-GTPase family is effective in psoriatic disease through regulation of IL-17/23/10, but not IL- 6 and TNFa.
Table 1. Demographics of psoriatic arthritis (PsA) patients.

\begin{tabular}{lll}
\hline & IL-17Ai & TNFi \\
\hline Number of PsA patients & 20 & 20 \\
Gender (\% female) & $55 \%$ & $75 \%$ \\
Mean Age (Years) & $55.9(9.5)$ & $56.8(7.9)$ \\
Mean Disease Duration (Years) & $10.4(6.9)$ & $7.3(7.9)$ \\
Mean DAPSA baseline & $38.8(17.5)$ & $45.6(28.9)$ \\
Responders (\%) (DAPSA <14) & $35 \%$ & $65 \%$ \\
Biologic naïve (\%) & $40 \%$ & $60 \%$ \\
\hline
\end{tabular}

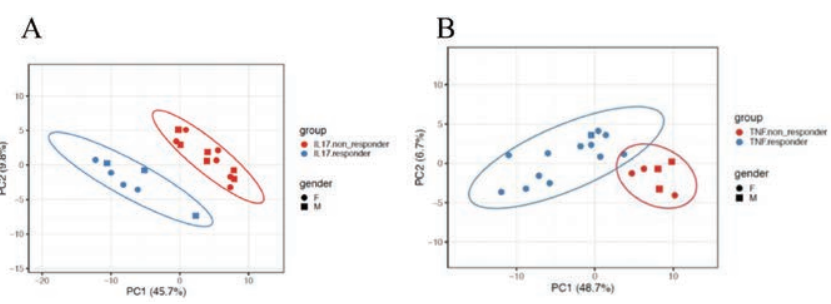

Figure 1. PCA plots illustrating that DEGs of $C D 4+$ cells can differentiate responders from non-responders when treated with: A. IL-17Ai; and B. TNFi.
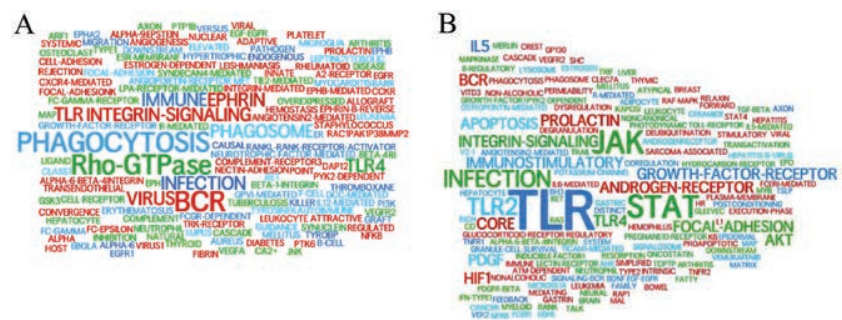

Figure 2. Over-represented terms in significantly enriched pathways considering DEGs between: A. IL-17i responders and non-responders (125 pathways); B. TNFi responders and non-responders (576 pathways)

Conclusion: Integration of cell-specific transcriptomic data with protein networks represents a very promising strategy for identifying biologic responders and pathways involved in predicting response that may have identified the RhoGTP pathway as a potential marker to guide the choice of biologic agents for individual patients.

Disclosure of Interests: Sara Rahmati: None declared, Darren O'Rielly: None declared, Quan Li: None declared, Dianne Codner: None declared, Amanda Dohey: None declared, Kari Jenkins: None declared, Igor Jurisica Grant/research support from: IBM, Dafna D Gladman Grant/research support from: AbbVie, Amgen Inc., BMS, Celgene Corporation, Janssen, Novartis, Pfizer, UCB - grant/ research support, Consultant of: AbbVie, Amgen Inc., BMS, Celgene Corporation, Janssen, Novartis, Pfizer, UCB - consultant, Vinod Chandran Grant/ research support from: Abbvie, Celgene, Consultant of: Abbvie, Amgen, Bris tol-Myers Squibb, Celgene, Eli Lily, Janssen, Novartis, Pfizer, UCB, Employee of: Spouse employed by Eli Lily, Proton Rahman Grant/research support from: Janssen and Novartis, Consultant of: Abbott, AbbVie, Amgen, BMS, Celgene Lilly, Janssen, Novartis, and Pfizer., Speakers bureau: Abbott, AbbVie, Amgen, BMS, Celgene, Lilly, Janssen, Novartis, Pfizer

DOI: 10.1136/annrheumdis-2020-eular.2317

\section{Newly identified cell subsets driving disease pathogenesis}

OP0306
CD11C-SPECIFIC ABLATION OF SHP1 INDUCES
AUTOIMMUNE SIALADENITIS SIMILAR TO SJÖGREN'S
SYNDROME

M. Kinoshita ${ }^{1}$, Y. Kaneko ${ }^{2}$, M. Watanabe ${ }^{2}$, Y. Imai ${ }^{2}$, S. Shrestha ${ }^{2}$, J. Suwa ${ }^{2}$, Y. Ohishi ${ }^{2}$, H. Hamatani ${ }^{2}$, M. Nakasatomi ${ }^{2}$, T. Sakairi ${ }^{2}$, H. Ikeuchi ${ }^{2}$, Y. Nojima ${ }^{2}$, K. Hiromura ${ }^{2}{ }^{1}$ Gunma University Graduate School of Medicine, Department of Nephrology and Rheumatology, Maebashi, Japan; ${ }^{1}$ Gunma University Graduate School of Medicine, Department of Nephrology and Rheumatology, Maebashi, Japan 
Background: Dendritic cells (DCs) play important roles in inducing immune response as well as maintaining immune tolerance. Src homology 2 domain-containing protein tyrosine phosphatase-1 (Shp1) is a negative regulator of signaling in hematopoietic cells and is expressed in a variety of immune cells including DCs. Shp1 homozygous mutant mice (motheaten mice) develop multiple immunological abnormalities and they die around four weeks after birth because of severe pneumonitis. Motheaten mice produce large amounts of autoantibodies, and besides, B-1a cells, a distinct B cell subset, which are an important source of autoantibodies increase in these mice. The functional abnormality of DCs in motheaten mice has not been characterized, but DCs and macrophages increase in various organs of motheaten mice.

To analyze the function of Shp1 in DCs, we generated Shp1 conditional knockout

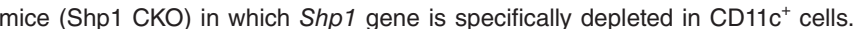
We found that aged shp1 CKO developed autoimmune glomerulonephritis. We also found that they developed severe tubulointerstitial nephritis (TIN) at the age of 40 weeks, which is characterized by the infiltration of $\mathrm{CD} 11 \mathrm{c}^{+}$and $\mathrm{F} 4 / 80^{+}$cells. $\mathrm{CD}^{+} \mathrm{T}$ cells from Shp1 CKO produce much more amount of IFN $\gamma$. Collectively, Shp1 in DCs acts as a key regulatory molecule to protect against autoimmunity. Objectives: We analyzed salivary glands of CKO to confirm whether they have autoimmune sialadenitis because TIN is known to be the most common renal manifestations of Sjögren's syndrome in human.

Methods: Shp1 CKO are generated by crossing a mouse line carrying floxed Shp1 allele to mice expressing Cre recombinase under the control of the CD11c promoter. Sex- and age-matched Ptpn6 ${ }^{\mathrm{fl} / \mathrm{fl}}$ littermates without Cre gene were studied as controls. We analyzed secretory function of the salivary glands in response to pilocarpine stimulation in Shp1 CKO at the age of 40 weeks or older. We then performed histological examination of salivary glands (submandibular glands and sublingual glands) with light-microscopy and immunohistochemical staining. The mononuclear cells prepared from the salivary glands were analyzed by flow cytometry (FCM). We also quantified anti-SSA/Ro60 antibodies and antiSSB/LA antibodies by ELISA.

Results: Shp1 CKO secreted less saliva flow compared to control mice by pilocarpine stimulation. Histological study showed Shp1 CKO exhibited massive infiltration of inflammatory cells in salivary glands associated with periductal foci and periductal fibrosis. Most of infiltrated cells were stained by anti- CD4 or B220 mAbs. FCM revealed that B cells increased in the salivary glands of Shp1 CKO. In addition, B-1a cells also increased in the salivary glands of the mice. The levels of anti-SSA/Ro60 antibodies and anti-SSB/LA antibodies were increased in Shp1 CKO.

Conclusion: CD11c-specific ablation of Shp1 induces the ectopic generation of lymphoid structure in salivary glands and impairment of salivary secretion. Autoantibody profile in Shp1 CKO resembled that in human Sjögren's syndrome. Our findings suggest that aged Shp1 CKO have the potential to become a new mouse model for the analysis of Sjögren's syndrome.

References:

[1] Green C. M. et al. J Heredity. 1975; 250-258.

[2] Kaneko T. et al. J Immunology. 2012; 5397-540.

[3] Watanabe M. et al. Biochem Biophys Rep. in press.

Disclosure of Interests: Masato Kinoshita: None declared, Yoriaki Kaneko Grant/research support from: CHUGAI PHARMACEUTICAL CO., LTD.

Astellas Pharma Inc. b, Speakers bureau: CHUGAI PHARMACEUTICAL CO., LTD. Astellas Pharma Inc., Mitsuharu Watanabe: None declared, Yoichi Imai: None declared, Shreya Shrestha: None declared, Junya Suwa: None declared, Yuko Ohishi: None declared, Hiroko Hamatani: None declared, Masao Nakasatomi: None declared, Toru Sakairi: None declared, Hidekazu Ikeuchi Speakers bureau: CHUGAI PHARMACEUTICAL CO., LTD.

Astellas Pharma Inc., Yoshihisa Nojima: None declared, Keiju Hiromura Grant/ research support from: CHUGAI PHARMACEUTICAL CO., LTD.

Astellas Pharma Inc., Speakers bureau: CHUGAI PHARMACEUTICAL CO., LTD. Astellas Pharma Inc.

DOI: 10.1136/annrheumdis-2020-eular.1044

\section{OP0307 A NOVEL TARGETED APPROACH TO ACHIEVE IMMUNE SYSTEM RESET: CD45-TARGETED ANTIBODY DRUG CONJUGATES AMELIORATE DISEASE IN PRECLINICAL AUTOIMMUNE DISEASE MODELS AND ENABLE AUTO-HSCT}

G. Gillard ${ }^{1}$, J. Proctor ${ }^{1}$, S. Hyzy ${ }^{1}$, O. Mikse ${ }^{1}$, T. Lamothe ${ }^{1}$, S. Mcdonough ${ }^{1}$ N. Clark', R. Palchaudhuri ${ }^{1}$, A. Bhat ${ }^{1}$, M. Brooks ${ }^{1}$, G. Sarma ${ }^{1}$, P. Bhattarai ${ }^{1}$, P. Sawant ${ }^{1}$, B. Pearse ${ }^{1}$, C. Mcdonagh ${ }^{1}$, T. Boitano ${ }^{1}$, M. Cooke ${ }^{1} .{ }^{1}$ Magenta Therapeutics, Cambridge, United States of America

Background: Resetting the immune system through autologous hematopoietic stem cell transplant (autoHSCT) is a highly effective treatment in selected patients with autoimmune diseases. AutoHSCT can induce long-term remission with $80 \%$ progression free survival in multiple sclerosis patients (Muraro 2017, Burt 2019). Use of autoHSCT in scleroderma patients has achieved superior outcomes in two randomized studies compared to standard of care (Tyndall 2014, Sullivan 2018). These impressive results are achieved by a combination of the eradication of autoreactive immune effector cells and re-establishment of self-tolerance, i.e., immune system reset. However, only a small fraction of eligible patients undergo autoHSCT, largely due to toxicity associated with current conditioning protocols.

Objectives: As part of our goal to enable more patients to benefit from immune system reset, we have generated novel anti-human CD45 ADCs that cross react with nonhuman primates (NHP) and an anti-mouse CD45 ADC to model the approach in mouse models of AID.

Methods: The human-targeted CD45-ADC is an affinity-matured mAb that targets an epitope present on all human CD45 isoforms, is cross-reactive with NHP CD45, and is conjugated to a payload that efficiently kills both quiescent and cycling cells. This ADC is engineered to eliminate Fc-mediated effector function, enable site-specific conjugation of linker/payload, and enable rapid clearance. This ADC was evaluated in vitro and in vivo in $\mathrm{hNSG}$ and NHPs. The murine too ADC specifically targets the CD45.2 isoform of mouse CD45, and is also engineered to eliminate effector function, allow for site-specific conjugation of linker payload, and be rapidly cleared. The payload for this murine tool ADC is potent and preferentially kills dividing cells. This ADC was tested for the ability to enable immune reset and ameliorate autoimmune disease in multiple disease models. Results: The anti-human CD45-ADC showed efficient killing of human HSCs and human and cyno PBMC, including $\mathrm{CD}^{+}$cells from healthy donors and patients with MS. In hNSG, single doses of the CD45-ADC were well-tolerated and led to substantial depletion of human cells. In NHPs, single doses of CD45-ADC were well tolerated and depleted both peripheral lymphocytes and HSCs. Administration of a single dose of anti-human CD45-ADC to hNSGs with sclerodermatous xenoGVHD resulted in depletion of human T cells and resolution of symptoms. A single-dose of the anti-mouse CD45-ADC enabled full myeloablation and complete durable donor chimerism with congenic HSCT at 16 weeks. In a murine immunization model of MS, MOG-induced EAE, a single dose of the CD45-ADC followed by congenic HSCT prior to disease onset enabled full donor chimerism, significantly delayed disease onset and reduced disease severity. We are generating additional data in an adoptive transfer model of EAE to confirm and extend these results. In a murine model of arthritis, therapeutic treatment with a single dose of the CD45-ADC followed by congenic HSCT enabled complete donor chimerism and halted disease progression, comparable to with the effects of an anti-TNFa antibody. The ADC is being further evaluated in a model of type 1 diabetes and those data will be presented. These data demonstrate that CD45-ADC conditioning followed by congenic HSCT is sufficient for full myeloablation and immune reset.

Conclusion: These results demonstrate that targeted immune depletion with a single dose of CD45-ADC can enable auto-HSCT and immune reset in multiple AID indications without toxic side effects. Targeted conditioning with CD45-ADC may represent a better tolerated approach for removing disease-causing cells as part of immune reset through auto-HSCT and enable more patients to benefit.

Figure 1. Anti-human CD45-ADC Depletes lymphocytes and HSCs at well tolerated doses in vivo in NHP

A
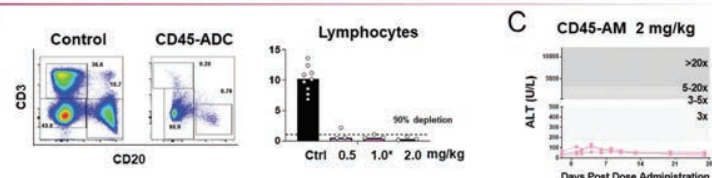

B

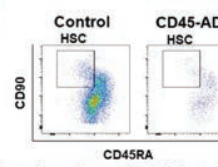
45-ADC
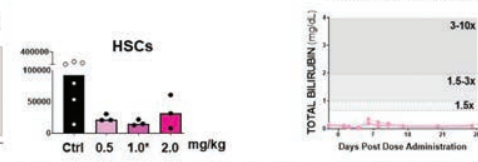

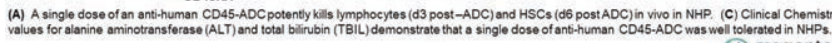

un magenta

Figure 2. CD45-ADCs Deplete Target Cells and Exert Potent Disease

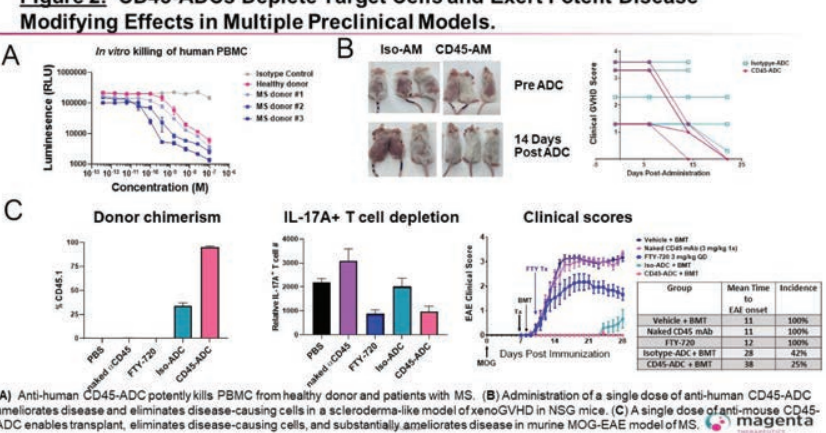

